\title{
MODULUS OF NEARLY UNIFORM SMOOTHNESS AND LINDENSTRAUSS FORMULAE
}

\author{
by TOMÁS DOMÍNGUEZ BENAVIDES $\dagger$
}

(Received 20 July, 1993)

Abstract. The Lindenstrauss formula

$$
\rho_{X^{*}}(t)=\sup _{0 \leq \epsilon \leq 2} \frac{t \epsilon}{2}-\delta_{X}(\epsilon)
$$

which states a strong relationship between the (Clarkson) modulus of uniform convexity $\delta_{X}$ of a Banach space $X$ and the modulus of uniform smoothness $\rho_{X^{*}}$ of the conjugate space $X^{*}$, is well known. Following the idea of the definitions of nearly uniform smooth space by S. Prus and modulus of uniform smoothness we define a modulus of nearly uniform smoothness and prove some Lindenstrauss type formulae concerning this modulus and the modulus of nearly uniform convexity for some measures of noncompactness.

1. Introduction and notation. Let $X$ be a Banach space. We denote by $B_{X}=$ $\{x \in X:\|x\| \leq 1\}$ the closed unit ball of $X$, by $S_{X}=\{x \in X:\|x\|=1\}$ the unit sphere and by $X^{*}$ the conjugate space of $X$. The notion of uniform convexity has been very useful in the geometric theory of Banach spaces and fixed point theory. We recall that $X$ is said to be uniformly convex (U.C.) if for every $\epsilon>0$ there exists $\delta>0$ such that $\|x+y\| / 2 \leq$ $1-\delta$ if $x$ and $y$ are in $B_{X}$ and $\|x-y\| \geq \epsilon$. The function

$$
\delta_{X}(\epsilon)=\inf \left\{1-\frac{\|x+y\|}{2}: x, y \in B_{X},\|x-y\| \geq \epsilon\right\}
$$

is called the (Clarkson) modulus of uniform convexity and the number $\epsilon_{0}(X)=\sup \{\epsilon \geq 0$ : $\left.\delta_{X}(\epsilon)=0\right\}$ is called the characteristic of convexity of $X$. It is clear that $X$ is U.C. if and only if $\epsilon_{0}(X)=0$. The most important generalization of the concept of uniform convexity in the fixed point theory is the notion of nearly uniform convexity. We recall [9] that $X$ is said to be nearly uniform convex (N.U.C.) if for every $\epsilon>0$ there exists $\delta>0$ such that $\inf \{\|x\|: x \in A\} \leq 1-\delta$ for every convex subset $A$ of $B_{X}$ such that $\mu(A) \geq \epsilon$, where $\mu$ is a measure of noncompactness. The function

$$
\Delta_{X, \mu}(\epsilon)=1-\sup \left\{\inf \|x\|: x \in A, A \text { convex subset of } B_{X} \text { with } \mu(A) \geq \epsilon\right\}
$$

is called the modulus of nearly uniform convexity of $X$ for the measure $\mu$. This modulus has been studied in [8] when $\mu=\alpha$ is the Kuratowski measure of noncompactness, i.e. $\alpha(A)=\inf \{\epsilon>0: A$ can be covered by finitely many sets with diameter less than $\epsilon\}, A$ being a bounded subset of $X$. For $\mu=\beta$, the Hausdorff measure of noncompactness, i.e.

$\dagger$ The research of the author is partially supported by the OGICYT (research project PB90-0903) and the Junta de Andalucia.

Glasgow Math. J. 37 (1995) 143-153. 
$\beta(A)=\inf \{\epsilon>0: A$ can be covered by finitely many balls with radii less than $\epsilon\}$ this modulus has been studied in [3]. For $\mu=\sigma$, the Istratescu measure of noncompactness, i.e. $\sigma(A)=\sup \{\epsilon>0$ : there exists an $\epsilon$-separated sequence in $A\}$, this modulus has been studied in [7], where it is proved that, for reflexive spaces, $\Delta_{X, \sigma}$ coincides with the Partington coefficient [10] of the uniform Kadec-Klee property, i.e. $P(\epsilon)=1-\sup \{\|x\|$ : $\left\{x_{n}\right\}$ is a $\epsilon$-separated sequence in $B_{X}$ converging weakly to $\left.x\right\}$. The characteristics of convexity $\epsilon_{0, \alpha}(X), \epsilon_{0, \beta}(X), \epsilon_{0, \sigma}(X)$ are defined in a similar way as $\epsilon_{0}(X)$ and $X$ is N.U.C. if and only if $\epsilon_{0, \mu}(X)=0$ for $\mu=\alpha$ or $\mu=\beta$ or $\mu=\sigma$.

On the other hand the dual notion of uniform convexity is the concept of uniform smoothness. The space $X$ is said to be uniformly smooth (U.S.) if $\lim _{t \rightarrow 0} t^{-1} \rho_{X}(t)=0$, where

$$
\rho_{X}(t)=\sup \left\{\frac{\|x+t y\|+\|x-t y\|}{2}-1: x, y \in B_{X}\right\} .
$$

The function $\rho_{X}(t)$ is called the modulus of uniform smoothness. From the Lindenstrauss formula

$$
\rho_{X^{*}}(t)=\sup _{0 \leq \epsilon \leq 2}\left(\frac{t \epsilon}{2}-\delta_{X}(\epsilon)\right)
$$

it easily follows that $X$ is U.C. if and only if $X^{*}$ is U.S. Having in mind the above duality property and the utility of the concept of uniform smoothness in the fixed point theory it is natural that several authors tried to define nearly uniformly smooth (N.U.S.) spaces. In $[13,4]$. N.U.S. spaces are defined as the conjugate spaces of N.U.C. spaces (in the reflexive case in [4]). A clearly different definition (but equivalent to the definition in [13]) is the following, given in [12]. (We recall that a sequence $\left\{x_{n}\right\}$ in a Banach space is called a basic sequence if it is a Schauder basis for the space which is spanned by $\left\{x_{n}\right\}$.)

Definition. A Banach space $X$ is said to be N.U.S. if for every $\epsilon>0$ there exists $\eta>0$ such that for every $t \in[0, \eta)$ and every basic sequence $\left\{x_{n}\right\}$ in $B_{X}$ there exists $k \in N$ such that $\left\|x_{1}+t x_{k}\right\| \leq \epsilon t+1$.

In this paper we define a modulus of nearly uniform smoothness (using the definition of U.S. spaces and of N.U.S. spaces in [12]). We prove some Lindenstrauss type inequalities for the modulus of nearly uniform smoothness of $X^{*}$ and the modulus $\Delta_{X, \sigma}$. When $X$ satisfies an Opial condition, we also prove Lindenstrauss inequalities with the modulus $\Delta_{X, \beta}$. Finally we list some open questions concerning fixed point theory and N.U.S. spaces.

\section{The modulus of nearly uniform smoothness.}

Definition 1. Let $X$ be a Banach space. We define the modulus of nearly uniform smoothness of $X$ as the function

$$
\Gamma_{X}(t)=\sup \left\{\inf \left\{\frac{\left\|x_{1}+t x_{n}\right\|+\left\|x_{1}-t x_{n}\right\|}{2}-1: n>1\right\}:\left\{x_{n}\right\} \text { basic sequence in } B_{X}\right\} .
$$

It is obvious from the definition that $\rho_{X}(t) \geq \Gamma_{X}(t)$ for every $t \geq 0$. Then if $X$ is U.S. 
we obtain $\lim _{t \rightarrow 0} \frac{\Gamma_{X}(t)}{t}=0$. We shall give an equivalent definition for $\Gamma_{X}(t)$ when $X$ is a reflexive space.

Proposition 1. Let $X$ be a reflexive Banach space. Then

$$
\Gamma_{X}(t)=\sup \left\{\inf \left\{\frac{\left\|x_{1}+t x_{n}\right\|+\left\|x_{1}-t x_{n}\right\|}{2}-1: n>1\right\}:\left\{x_{n}\right\} \text { weakly null in } B_{X}\right\} .
$$

Proof. Let

$$
\Gamma_{X}^{\prime}(t)=\sup \left\{\inf \left\{\frac{\mid x_{1}+t x_{n}\|+\| x_{1}-t x_{n} \|}{2}-1: n>1\right\}:\left\{x_{n}\right\} \text { weakly null in } B_{X}\right\} .
$$

Since $X$ is reflexive, every basic sequence is weakly null. Thus $\Gamma_{X}^{\prime}(t) \geq \Gamma_{X}(t)$. On the other hand, let $\left\{x_{n}\right\}$ be a weakly null sequence in $B_{X}$. If $\lim \inf \left\|x_{n}\right\|>0$ there exists a basic subsequence $\left\{y_{n}\right\}$ of $\left\{x_{n}\right\}$ such that $y_{1}=x_{1}$. Thus

$$
\begin{aligned}
\Gamma_{X}(t) & \geq \inf \left\{\frac{\left\|y_{1}+t y_{n}\right\|+\left\|y_{1}-t y_{n}\right\|}{2}-1: n>1\right\} \\
& \geq \inf \left\{\frac{\left\|x_{1}+t x_{n}\right\|+\left\|x_{1}-t x_{n}\right\|}{2}-1: n>1\right\} .
\end{aligned}
$$

If $\lim \inf \left\|x_{n}\right\|=0$ there exists a subsequence $\left\{y_{n}\right\}$ of $\left\{x_{n}\right\}$ such that $y_{1}=x_{1}$ and $\lim y_{n}=0$. Let $\eta$ be an arbitrary number bigger than $\Gamma_{X}(t)$. There exists an integer $n_{0}$ such that $\left\|y_{n}\right\|<\eta / t$ if $n \geq n_{0}$. Then

$$
\begin{aligned}
\eta \geq \frac{\left\|y_{1}+t y_{n}\right\|+\left\|y_{1}-t y_{n}\right\|}{2}-1 & \geq \inf \left\{\frac{\left\|y_{1}+t y_{n}\right\|+\left\|y_{1}-t y_{n}\right\|}{2}-1: n>1\right\} \\
& \leq \inf \left\{\frac{\left\|x_{1}+t x_{n}\right\|+\left\|x_{1}-t x_{n}\right\|}{2}-1: n>1\right\} .
\end{aligned}
$$

By (1) and (2) we obtain $\eta \geq \Gamma_{X}^{\prime}(t)$. Thus $\Gamma_{X}^{\prime}(t) \leq \Gamma_{X}(t)$. and

Proposition 2. Let $X$ be a Banach space. Then $X$ is N.U.S. if and only if $X$ is reflexive

$$
\lim _{t \rightarrow 0} t^{-1} \Gamma_{X}(t)=0
$$

Proof. If $\lim _{t \rightarrow 0} t^{-1} \Gamma_{X}(t)=0$, for every $\epsilon>0$ there exists $\eta>0$ such that $\Gamma_{X}(t) \leq t \epsilon$ for $t \in[0, \eta]$. Let $\left\{x_{n}\right\}$ be a basic sequence in $B_{X}$. Since $X$ is reflexive, a similar argument as in [12, after definition 2.1] proves that we can assume that $\left\{x_{n}\right\}$ is not norm convergent and

$$
\left\|x_{1}+t x_{n_{k}}\right\| \leq \frac{1}{2}\left((1+c)\left\|x_{1}-2 t x_{n_{k}}\right\|+\left\|x_{1}+2 t x_{n_{k}}\right\|\right),
$$


where $c>1,1+c<(1+3 t \epsilon) /(1+2 t \epsilon)$ and $\left\{x_{n_{k}}\right\}$ is a subsequence of $\left\{x_{n}\right\}$. Then for some $k$ we have

$$
\left\|x_{1}+t x_{n_{k}}\right\| \leq(1+c)(1+2 t \epsilon)<1+3 t \epsilon .
$$

Conversely if $X$ is N.U.S., then $X$ is reflexive. Let $\left\{x_{n}\right\}$ be a weakly null sequence. Then for every $\epsilon>0$ there exists $\eta>0$ such that $\left\|x_{1}+t z_{n}\right\| \leq 1+\epsilon t$ for every $n>1$, where $\left\{z_{n}\right\}$ is a subsequence of $\left\{x_{n}\right\}$ with $z_{1}=x_{1}$. Since the sequence $\left\{x_{1},-z_{2},-z_{3}, \ldots\right\}$ is also weakly null we have $\left\|x_{1}-t z_{n}\right\| \leq 1+\epsilon t$ for some $n>1$ and every $t \in[0, \eta]$. Thus

$$
\left(\left\|x_{1}+t z_{n}\right\|+\left\|z_{1}-t z_{n}\right\|\right) / 2-1 \leq \epsilon t
$$

if $t \in[0, \eta]$. Hence $\lim _{t \rightarrow 0} t^{-1} \Gamma_{X}(t)=0$.

3. Lindenstrauss formulae. We shall prove some Lindenstrauss type inequalities for the modulus of nearly uniform smoothness and the modulus of nearly uniform convexity for the Istratescu measure of noncompactness, which can be considered as a quantitative version of Theorem 2.4 in [12]. We recall the definition of the weak normal structure coefficient given by [5].

$$
\begin{array}{r}
W C S(X)=\inf \left\{\frac{\operatorname{diam}_{a}\left\{x_{n}\right\}}{r_{a}\left\{x_{n}\right\}}:\left\{x_{n}\right\}\right. \text { is a weakly convergent sequence } \\
\text { which is not norm convergent }\},
\end{array}
$$

where $r_{a}\left\{x_{n}\right\}$ is the asymptotic radius of $\left\{x_{n}\right\}$; i.e. $\inf \left\{\lim \sup \left\|x_{n}-y\right\|: y \in \operatorname{co}\left\{x_{n}\right\}\right\}$ and $\operatorname{diam}_{a}\left\{x_{n}\right\}$ is the asymptotic diameter of $\left\{x_{n}\right\}$, i.e. $\operatorname{diam}_{a}\left\{x_{n}\right\}=\lim _{k} \sup _{n, m \geq k}\left\|x_{n}-x_{m}\right\|$. Using some results of [6] and [11], it is proved in [7] that for any reflexive space $X$ we have

$$
\begin{gathered}
W C S(X)=\inf \left\{\lim _{n, m ; n \neq m}\left\|x_{n}-x_{m}\right\|:\left\{x_{n}\right\}\right. \text { is a weakly null sequence, } \\
\left.\qquad \lim \left\|x_{n}\right\|=1 \text { and } \lim _{n, m ; n \neq m}\left\|x_{n}-x_{m}\right\| \text { exists }\right\} .
\end{gathered}
$$

We also recall that a Banach space $X$ is said to satisfy the Opial condition if

$$
\lim \inf \left\|x_{n}\right\| \leq \liminf \left\|x+x_{n}\right\|
$$

for each weakly null sequence $\left\{x_{n}\right\}$.

THEOREm 1. Let $X$ be a reflexive Banach space. Then

$$
\sup _{0 \leq \epsilon \leq a} \frac{t \epsilon W C S\left(X^{*}\right)}{4}-\Delta_{X, \sigma}(\epsilon) \leq \Gamma_{X^{*}}(t) \leq \sup _{0 \leq \epsilon \leq a} \frac{t \epsilon}{W C S(X)}-\Delta_{X, \sigma}(\epsilon)
$$

for every $t>0$, where $a=\sigma\left(B_{X}\right)$. If, in addition, $X^{*}$ satisfies the Opial condition, then

$$
\sup _{0 \leq \epsilon \leq a} \frac{t \epsilon}{2}-\Delta_{X, \sigma}(\epsilon) \leq \Gamma_{X^{*}}(t)
$$


Proof. (1) Let $\left\{x_{n}\right\}$ be a sequence in $B_{X}$ converging weakly to $x$, such that $\left\|x_{n}-x_{m}\right\| \geq \epsilon$ for every $n, m, n \neq m$. Choose $f \in S_{X^{*}}$ such that $f(x)=\|x\|$ and for a given $\eta>0$ choose an integer $N$ such that $\left|f\left(x_{n}\right)-\|x\|\right|<\eta$ if $n \geq N$. Choose $g_{n} \in S_{X^{*}}$ such that $g_{n}\left(x_{n}-x_{n+1}\right)=\left\|x_{n}-x_{n+1}\right\|$. By reflexivity there exists a subsequence $g_{n_{k}}$ converging weakly to $g$. We can also assume that there exists $\lim \left\|g_{n_{k}}-g\right\|$. For $N_{1}$ large enough we have $\left|g\left(x_{n}\right)-g\left(x_{n+1}\right)\right|<\eta$ if $n>N_{1}$. Denote $h_{n_{1}}=f$ and for $k \geq 2, h_{n_{k}}=c^{-1}\left(g_{n_{k}}-g\right)$, where $c=1$ if $X^{*}$ satisfies the Opial condition; otherwise $c=(2+\eta) / W C S\left(X^{*}\right)$. Since

$$
W C S\left(X^{*}\right) \leq \frac{\operatorname{diam}_{a}\left\|g_{n_{k}}-g_{n_{j}}\right\|}{\lim \left\|g_{n_{k}}-g\right\|} \leq \frac{2}{\lim \left\|g_{n_{k}}-g\right\|}
$$

we have that $\left\{h_{n_{k}}\right\}$ belongs to $B_{X^{*}}$ for $k$ large enough either if $X^{*}$ satisfies the Opial condition or if it does not. Since $h_{n_{k}}$ is weakly null we have

$$
\Gamma_{X^{*}}(t) \geq \inf \left\{\frac{\left\|h_{n_{1}}+t h_{n_{k}}\right\|+\left\|h_{n_{1}}-t h_{n_{k}}\right\|}{2}-1: k \geq 2\right\}
$$

Hence, there exists an integer $k$ such that

$$
\begin{aligned}
\Gamma_{X^{*}}(t)+\eta & \geq \frac{1}{2}\left(\left\|h_{n_{1}}+t h_{n_{k}}\right\|+\left\|h_{n_{1}}-t h_{n_{k}}\right\|\right)-1 \\
& \geq \frac{1}{2}\left(h_{n_{1}}+t h_{n_{k}}\right)\left(x_{n_{k}}\right)+\frac{1}{2}\left(h_{n_{1}}-t h_{n_{k}}\right)\left(x_{n_{k}+1}\right)-1 \\
& =\frac{1}{2} h_{n_{1}}\left(x_{n_{k}}+x_{n_{k}+1}\right)+\frac{1}{2} t h_{n_{k}}\left(x_{n_{k}}-x_{n_{k}+1}\right)-1 \\
& \geq \frac{1}{2}(2\|x\|-2 \eta)+\frac{1}{2 c} t\left\|x_{n_{k}}-x_{n_{k}+1}\right\|-\frac{t \eta}{2 c}-1 \\
& \geq\|x\|+\frac{1}{2 c} t \epsilon-2 \eta-t \eta-1 .
\end{aligned}
$$

Thus

$$
1-\|x\| \geq \frac{t \epsilon}{2 c}-\Gamma_{X^{*}}(t)-2 \eta-t \eta
$$

Since $\left\{x_{n}\right\}$ is an arbitrary sequence with $\sigma\left(\left\{x_{n}\right\}\right) \geq \epsilon$ we have

$$
\Delta_{X_{, \sigma}}(\epsilon) \geq \frac{t \epsilon}{2 c}-\Gamma_{X^{*}}(t)-2 \eta-t \eta
$$

Since $\eta>0$ and $0 \leq \epsilon \leq a$ are arbitrary we obtain

$$
\Gamma_{X^{*}}(t) \geq \sup _{0 \leq \epsilon \leq a} \frac{t \epsilon W C S\left(X^{*}\right)}{4}-\Delta_{X, \sigma}(\epsilon),
$$

if $X^{*}$ does not satisfy the Opial condition. Otherwise

$$
\Gamma_{X^{*}}(t) \geq \sup _{0 \leq \epsilon \leq a} \frac{t \epsilon}{2}-\Delta_{X, \sigma}(\epsilon)
$$

(2) Let $\left\{x_{n}^{*}\right\}$ be a weakly null sequence in $B_{X^{*}}$. Choose $x_{n} \in S_{X}(n \geq 2)$ such that $\left\|x_{1}^{*}+t x_{n}^{*}\right\|=\left(x_{1}^{*}+t x_{n}^{*}\right)\left(x_{n}\right)$. Taking a subsequence we can assume that $\left\{x_{n}\right\}$ converges weakly to $x$. Denote $\epsilon=\sigma\left(\left\{x_{n}\right\}\right)$. Taking again a subsequence, we can also assume 
$\epsilon \leq\left\|x_{n}-x_{m}\right\| \leq \epsilon+\eta$ for an arbitrary positive number $\eta$. Choose $\eta$ large enough such that $\left|x_{1}^{*}\left(x_{n}\right)-x_{1}^{*}(x)\right|<\eta,\left|x_{n}^{*}(x)\right|<\eta$. Then

$$
\begin{aligned}
\left\|x_{1}^{*}+t x_{n}^{*}\right\|-1 & =x_{1}^{*}\left(x_{n}\right)+t x_{n}^{*}\left(x_{n}\right)-1 \\
& \leq x_{1}^{*}(x)+t x_{n}^{*}\left(x_{n}-x\right)+(1+t) \eta-1 \\
& \leq\|x\|+t\left\|x_{n}-x\right\|+(1+t) \eta-1 \\
& \leq\|x\|+t \frac{\operatorname{diam}\left(\left\{x_{n}\right\}\right)}{W C S(X)}+(1+t) \eta-1 \\
& \leq\|x\|+\frac{t \epsilon}{W C S(X)}+(1+2 t) \eta-1 \\
& \leq \frac{t \epsilon}{W C S(X)}+(1+2 t) \eta-\Delta_{X, \sigma}(\epsilon) .
\end{aligned}
$$

The same argument for $\left\|x_{1}^{*}-t x_{n}^{*}\right\|$ proves that

$$
\left\|x_{1}^{*}-t x_{n}^{*}\right\|-1 \leq \frac{t \epsilon^{\prime}}{W C S(X)}+(1+2 t) \eta-\Delta_{X, \sigma}\left(\epsilon^{\prime}\right)
$$

for some $\epsilon^{\prime} \leq a$. Thus

$$
\begin{aligned}
\frac{\left\|x_{1}^{*}+t x_{n}^{*}\right\|+\left\|x_{1}^{*}-t x_{n}^{*}\right\|}{2}-1 & \leq \frac{t \epsilon}{2 W C S(X)}+(1+2 t) \eta-\frac{\Delta_{X, \sigma}(\epsilon)}{2}+\frac{t \epsilon^{\prime}}{2 W C S(X)}-\frac{\Delta_{X, \sigma}\left(\epsilon^{\prime}\right)}{2} \\
& \leq \sup _{0 \leq \epsilon \leq a} \frac{t \epsilon}{W C S(X)}-\Delta_{X, \sigma}(\epsilon)+(1+2 t) \eta .
\end{aligned}
$$

Since $\eta$ is arbitrary we obtain (2).

Remark. The inclusion of the coefficient $\operatorname{WCS}(X)$ in the Lindenstrauss formula may be surprising. However we note that the function $\Gamma_{X^{*}}(t)$ is independent of any measure of noncompactness, whereas, $\Delta_{X, \mu}(\epsilon)$ depends on the measure of noncompactness which we are considering. The following example shows that when we use the Istratescu measure $W C S(X)$ cannot be removed in the inequality on the right side. However, Theorem 2 will show that for the Hausdorff measure of noncompactness, we can obtain in the right side an expression similar to that in the classical Lindenstrauss formula.

EXAMPLE. Let $X$ be the Hilbert space $\ell^{2}$. If $\left\{x_{n}\right\}$ is a weakly null sequence, taking a subsequence $\left\{y_{n}\right\}$ with $y_{1}=x_{1}$, it can be proved that we can assume supp $y_{n} \cap \operatorname{supp} y_{m}=\varnothing$, where supp $y_{n}=\left\{k \in N: y_{n}, e_{k} \neq 0\right\}$. Considering a block basis we can also assume $y_{n}=e_{n}$. Since

$$
\frac{\left\|e_{1}+t e_{k}\right\|+\left\|e_{1}-t e_{k}\right\|}{2}-1=\sqrt{1+t^{2}}-1
$$

we have $\Gamma_{X}(t)=\sqrt{1+t^{2}}-1$. On the other hand $\Delta_{X, \sigma}(\epsilon)=1-\sqrt{1-\epsilon^{2} / 2}$. Hence

$$
\frac{t \epsilon}{W C S(X)}-\Delta_{X, \sigma}(\epsilon)=\frac{t \epsilon}{\sqrt{2}}-1+\sqrt{1-\frac{\epsilon^{2}}{2}} \text {. }
$$


A standard argument shows that

$$
\sup _{0 \leq \epsilon \leq \sqrt{2}} \frac{t \epsilon}{\sqrt{2}}-1+\sqrt{1-\frac{\epsilon^{2}}{2}}=\sqrt{1+t^{2}}-1 .
$$

Thus

$$
\Gamma_{X^{*}}(t)=\sup _{0 \leq \epsilon \leq \sqrt{2}} \frac{t \epsilon}{W C S(X)}-\Delta_{X, \sigma}(\epsilon)
$$

for every $t>0$.

REMARK. It is clear from the Lindenstrauss formula that the function $\rho_{X}(t) / t$ is non-decreasing. The same result can be directly proved for $\Gamma_{X}(t) / t$. Indeed, for every sequence $\left\{x_{n}\right\}$ and all positive numbers $t, s$ with $t<s$ we can choose $h_{n}, g_{n} \in S_{X^{*}}$ such that $h_{n}\left(x_{1}+t x_{n}\right)=\left\|x_{1}+t x_{n}\right\|$ and $g_{n}\left(x_{1}-t x_{n}\right)=\left\|x_{1}-t x_{n}\right\|$. Then

$$
\begin{aligned}
\frac{\left\|x_{1}+t x_{n}\right\|+\left\|x_{1}-t x_{n}\right\|-2}{2 t} & =\frac{h_{n}\left(x_{1}+t x_{n}\right)+g_{n}\left(x_{1}-t x_{n}\right)-2}{2 t} \\
& =\frac{\left(h_{n}+g_{n}\right)\left(x_{1}\right)-2}{2 t}+\frac{\left(h_{n}-g_{n}\right)\left(x_{n}\right)}{2} .
\end{aligned}
$$

Since $\left|\left(h_{n}+g_{n}\right)\left(x_{1}\right)\right| \leq 2$ we have

$$
\frac{\left(h_{n}+g_{n}\right)\left(x_{1}\right)-2}{2 t} \leq \frac{\left(h_{n}+g_{n}\right)\left(x_{1}\right)-2}{2 s}
$$

Thus

$$
\frac{\left\|x_{1}+t x_{n}\right\|+\left\|x_{1}-t x_{n}\right\|-2}{2 t} \leq \frac{\left\|x_{1}+s x_{n}\right\|+\left\|x_{1}-s x_{n}\right\|-2}{2 s}
$$

COROLlary. Let $X$ be a reflexive Banach space. Then

$$
W C S(X) \lim _{t \rightarrow 0} \frac{\Gamma_{X^{*}}(t)}{t} \leq \epsilon_{0, \sigma}(X) \leq \frac{4}{W C S\left(X^{*}\right)} \lim _{t \rightarrow 0} \frac{\Gamma_{X^{*}}(t)}{t}
$$

Proof. Denote $a=\lim _{t \rightarrow 0} t^{-1} \Gamma_{X^{*}}(t)$. For every $\eta>0$ and $t$ small enough we have

$$
\frac{a+\eta}{2} \geq \frac{\epsilon W C S\left(X^{*}\right)}{4}-\frac{\Delta_{X, \sigma}(\epsilon)}{t}
$$

for every $\epsilon$. Thus $\Delta_{X, \sigma}(\epsilon)>0$ for every $\epsilon>4 a / W C S\left(X^{*}\right)+\eta$. Since $\eta$ is arbitrary we have $\epsilon_{0, \sigma}(X) \leq 4 a / W C S\left(X^{*}\right)$. On the other hand for any $\eta>0$ and $t$ small enough we have

$$
\sup _{0 \leq \epsilon \leq a} \frac{\epsilon}{W C S(X)}-\frac{\Delta_{X, \sigma}(\epsilon)}{t}>a-\eta
$$


Thus for some $\epsilon(t)$ we have

$$
\frac{\epsilon(t)}{W C S(X)}-\frac{\Delta_{X, \sigma}(\epsilon(t))}{\epsilon_{0, \sigma}(X) t}>a-\eta
$$

Hence $\epsilon(t)>(a-\eta) W C S(X)$. Furthermore if $c=\Delta_{X, \sigma}((a-\eta) W C S(X))>0$, then $\Delta_{X, \sigma}(\epsilon(t))>c$ for every $t$. Letting $t \rightarrow 0$ we obtain $a-\eta<-\infty$. Thus $\Delta_{X, \sigma}((a-\eta) W C S(X))=0$ and $\epsilon_{0, \sigma}(X) \geq a W C S(X)$.

When $X$ is a Banach space satisfying the Opial condition we can obtain Lindenstrauss inequalities using the Hausdorff measures of noncompactness.

THEOREM 2. Let $X$ be a reflexive Banach space satisfying the Opial condition. Then

$$
\sup _{0 \leq \epsilon \leq 1} \frac{t \epsilon W C S(X) W C S\left(X^{*}\right)}{4}-\Delta_{X, \beta}(\epsilon) \leq \Gamma_{X^{*}}(t) \leq \sup _{0 \leq \epsilon \leq t} t \epsilon-\Delta_{X, \beta}(\epsilon)
$$

for every $t>0$. If , in addition, $X^{*}$ satisfies the Opial condition

$$
\sup _{0 \leq \epsilon \leq 1} \frac{t \epsilon W C S(X)}{2}-\Delta_{X, \beta}(\epsilon) \leq \Gamma_{X^{*}}(t)
$$

Proof. Let $\left\{x_{n}^{*}\right\}$ be a weakly null sequence in $B_{X^{*}}$. Choose $x_{n} \in S_{X}, n \geq 2$ such that $\left\|x_{1}^{*}+t x_{n}^{*}\right\|=\left(x_{1}^{*}+t x_{n}^{*}\right)\left(x_{n}\right)$. Taking a subsequence and using Lemma 2.6 in [2] we can assume that $\left\{x_{n}\right\}$ is $\beta$-minimal (see [6] for definitions) and $\epsilon=\beta\left(\left\{x_{n}\right\}\right)=\lim \left\|x_{n}-x\right\|$, where $x$ is the weak limit of $\left\{x_{n}\right\}$. The argument used in the proof of Theorem 1 shows that for an arbitrary $\eta>0$ we have

$$
\begin{aligned}
\left\|x_{1}^{*}+t x_{n}^{*}\right\|-1 & \leq\|x\|+t\left\|x_{n}-x\right\|+(1+t) \eta-1 \\
& \leq\|x\|+t \epsilon+(2 t+1) \eta-1 \\
& \leq t \epsilon-\Delta_{X, \beta}(\epsilon)+(2 t+1) \eta
\end{aligned}
$$

for $n$ large enough. A similar argument for $\left\|x_{1}^{*}-t x_{n}^{*}\right\|-1$ lets us state that

$$
\Gamma_{X^{*}}(t) \leq \sup _{0 \leq \epsilon \leq 1} t \epsilon-\Delta_{X, \beta}(\epsilon)
$$

On the other hand, by Proposition 7 in [1], (separability can be replaced by Opial condition according to Lemma 2.6 in [2]), we have

$$
\Delta_{X, \beta}(\epsilon)=\inf \left\{1-\|x\|:\left\{x_{n}\right\} \in B_{X} \text { converges weakly to } x, \beta\left(\left\{x_{n}\right\}\right) \geq \epsilon \text { and }\left\{x_{n}\right\} \text { satisfies } P\right\},
$$

where $P$ is any property such that every sequence $\left\{x_{n}\right\}$ in $B_{X}$ has a subsequence satisfying $P$. Let $\left\{x_{n}\right\}$ be a sequence in $B_{X}$ such that $\left\{x_{n}\right\}$ converges weakly to $x, \lim \left\|x_{n}-x\right\|=$ $\beta\left(\left\{x_{n}\right\}\right) \geq \epsilon$, and $\lim _{n, m ; n \neq m}\left\|x_{n}-x_{m}\right\|$ exists. Choose $f \in S_{X^{*}}$ such that $f(x)=\|x\|$ and for every $n \geq 2$ choose $g_{n} \in S_{X^{*}}$ such that $g_{n}(x)=\left\|x_{n}-x_{n+1}\right\|$. Taking a subsequence we can assume that $\left\{g_{n}\right\}$ converges weakly to $g$. Denote $h_{1}=g_{1}$ and $h_{n}=\left(g_{n}-g\right) / c$ where $c=1$ if 
$X^{*}$ satisfies the Opial condition and $c=2 / W C S\left(X^{*}\right)$ otherwise. The argument in the proof of Theorem 1 shows that for any $\eta>0$ there exists an integer $k$ such that

$$
\dot{\Gamma}_{X^{*}}(t)+\eta \geq\|x\|+\frac{1}{2 c} t\left\|x_{k}-x_{k+1}\right\|-(2+t) \eta-1
$$

Assuming $k$ large enough we have $\left\|x_{k}-x_{k+1}\right\| \geq \operatorname{diam}_{a}\left(\left\{x_{n}\right\}\right)-\eta$. Thus

$$
\begin{aligned}
\Gamma_{X^{*}}(t) & \geq\|x\|+\frac{1}{2 c} t \operatorname{diam}_{a}\left(\left\{x_{n}\right\}\right)-(3+2 t) \eta-1 \\
& \geq\|x\|+\frac{1}{2 c} t W C S(X) \lim \left\|x_{n}-x\right\|-(3+2 t) \eta-1 \\
& \geq\|x\|-1+\frac{1}{2 c} t W C S(X) \epsilon-(3+2 t) \eta
\end{aligned}
$$

Thus

$$
1-\|x\| \geq \frac{t W C S(X) \epsilon}{2 c}-\Gamma_{X^{*}}(t)-(3+2 t) \eta .
$$

Since every sequence in $B_{X}$ has a subsequence satisfying $\lim \left\|x_{n}-x\right\|=\beta\left(\left\{x_{n}\right\}\right)$ and there exists $\lim _{n, m ; n \neq m}\left\|x_{n}-x_{m}\right\|$ (see proof of Lemma 1.1 in [7]) the above definitions of $\Delta_{X, \beta}(\epsilon)$ let us state that

$$
\Delta_{X, \beta}(\epsilon) \geq \frac{t W C S(X) \epsilon}{2 c}-\Gamma_{X^{*}}(t)-(3+2 t) \eta
$$

Since $\eta$ is arbitrary we obtain

$$
\Gamma_{X *}(t) \geq \frac{t W C S(X) \epsilon}{2 c}-\Delta_{X, \beta}(\epsilon)
$$

or

$$
\Gamma_{X^{*}}(t) \geq \frac{t W C S(X) \epsilon}{2}-\Delta_{X, \beta}(\epsilon)
$$

if $X^{*}$ satisfies the Opial condition.

Remark. Let $X$ be a reflexive Banach space with the Opial condition. If, in addition, $X^{*}$ has a Schauder symmetric basis $\left\{e_{n}\right\}$ (i.e. $\left\|\sum_{n \geq 1} a_{n} e_{n}\right\|=\left\|\sum_{n \geq 1} \pm a_{n} e_{n}\right\|$ ) then

$$
\Gamma_{X^{*}}(t)=\sup _{0 \leq \epsilon \leq a} t \epsilon-\Delta_{X, \beta}(\epsilon)
$$

Indeed, let $\left\{x_{n}\right\}$ a sequence in $B_{X}$ converging weakly to $x$ and such that $\beta\left(\left\{x_{n}\right\}\right)=$ $\lim \left\|x_{n}-x\right\|=\epsilon$. Choose $g_{n} \in S_{X^{*}}$ such that $g_{n}\left(x_{n}-x\right)=\left\|x_{n}-x\right\|$, and $f \in S_{X^{*}}$ such that $f(x)=\|x\|$. Assume that $\left\{g_{n}\right\}$ converges weakly to $g$ and denote $h_{n}=g_{n}-g$. Since $X^{*}$ has a symmetric basis, this space satisfies the Opial condition. Thus $h_{n}$ belongs to $B_{X^{*}}$ for $n$ large enough. Since $\left\{h_{n}\right\}$ is weakly null, the coordinate functional $e_{k}^{*}\left(h_{n}\right)$ converges to 0 for every $k \in \mathbb{N}$. Hence we can assume, without loss of generality, that $\operatorname{supp} f \cap \operatorname{supp} h_{n}=\varnothing$ 
for $n$ large enough. (Here we denote $\operatorname{supp} f=\left\{k \in N: e_{k}^{*}(f) \neq 0\right\}$.) Thus $\left\|f+t h_{n}\right\|=$ $\left\|f-t h_{n}\right\|$ and we obtain for $\eta$ arbitrary and $n$ large enough

$$
\begin{aligned}
\Gamma_{X^{*}}(t)+\eta & \geq\left\|f+t h_{n}\right\|-1 \geq\left(f-t h_{n}\right)\left(x_{n}\right)-1 \\
. & \geq f\left(x_{n}\right)+t h_{n}\left(x_{n}-x\right)-t \eta-1 \\
& \geq\|x\|-1+t \epsilon-(2 t+1) \eta .
\end{aligned}
$$

Since $\eta$ is arbitrary we have

$$
\Gamma_{X^{*}}(t) \geq \sup _{0 \leq \epsilon \leq 1} t \epsilon-\Delta_{X, \beta}(\epsilon)
$$

Then we obtain the equality

$$
\Gamma_{X^{*}}(t)=\sup _{0 \leq \epsilon \leq 1} t \epsilon-\Delta_{X, \beta}(\epsilon) .
$$

Thus changing the variable $\epsilon$ by $\epsilon^{\prime}=2 \epsilon$, i.e. if we consider diameters instead of radii in the definition of the Hausdorff measure, we obtain the expression

$$
\Gamma_{X^{*}}(t)=\sup _{0 \leq \epsilon^{\prime} \leq 2} \frac{t \epsilon^{\prime}}{2}-\Delta_{X, \beta}\left(\epsilon^{\prime}\right),
$$

which is quite similar to the classical Lindenstrauss formula.

If, in addition, $X$ has the $\beta$-property (see [6] for definitions) we have

$$
\Gamma_{X^{*}}(t)=\sup _{0 \leq \epsilon \leq W C S(X)} \frac{t \epsilon}{W C S(X)}-\Delta_{x, \sigma}(\epsilon)
$$

Indeed, we have by Lemma 1.1 in [7]

$$
\begin{gathered}
W C S(X)=\inf \left\{\lim _{n, m ; n \neq m}\left\|x_{n}-x_{m}\right\|:\left\{x_{n}\right\}\right. \text { is a weakly null sequence, } \\
\left.\lim \left\|x_{n}\right\|=1 \text { and } \lim _{n, m ; n \neq m}\left\|x_{n}-x_{m}\right\| \text { exists }\right\} .
\end{gathered}
$$

If $X$ has the $\beta$-property with constant $\mu$, this quotient is a constant equal to $2 / \mu$. Since $\beta=\mu \sigma / 2$ we have $\Delta_{X, \sigma}(2 \epsilon / \mu)=\Delta_{X, \beta}(\epsilon)=\Delta_{X, \sigma}(W C S(X) \epsilon)$. Thus

$$
\Gamma_{X^{*}}(t)=\sup _{0 \leq \epsilon \leq 1} t \epsilon-\Delta_{X, \beta}(\epsilon)=\sup _{0 \leq \epsilon \leq W C S(X)} \frac{t \epsilon}{W C S(X)}-\Delta_{X, \sigma}(\epsilon) .
$$

These conditions are satisfied, for instance, for every $\ell^{p}$ space $(1<p<\infty)$. Hence

$$
\Delta_{\ell^{q}}=\left(1+t^{q}\right)^{1 / q}=\sup _{0 \leq \epsilon \leq 2^{1 / p}} \frac{t \epsilon}{2^{1 / p}}-\Delta_{\ell^{p}, \sigma}(\epsilon)
$$

Open Questions. (1) Does $\lim _{t \rightarrow 0} \Gamma_{X}(t) / t<1 / 2$ imply reflexivity or the fixed point property? 
(2) In the general case, can we obtain Lindenstrauss formulae for $\Delta_{X, \mu(\epsilon)}$ and $\Gamma_{X^{*}}(t)$ with equalities?

\title{
REFERENCES
}

1. J. Arias de Reyna and T. Domínguez Benavides, On a measure of noncompactness in Banach spaces with Schauder basis, Bollettino. U.M.I. 7A (1993), 77-86.

2. J. M. Ayerbe and T. Domínguez Benavides, Connections between some Banach space coefficients concerning normal structure, J. Math. Anal. Appl. 172 (1993), 53-61.

3. J. Banas, On modulus of noncompact convexity and its properties, Canad. Math. Bull. 30 (1987), 186-192.

4. J. Banas, Compactness conditions in the geometric theory of Banach spaces, Nonlin. Anal. 16 (1991), 669-682. 427-435.

5. W. L. Bynum, Normal structure coefficients for Banach spaces, Pacific J. Math. 86 (1980),

6. T. Domínguez Benavides, Some properties of the set and ball measures of noncompactness and applications, J. London Math. Soc. 34(2) (1986), 120-128.

7. T. Domínguez Benavides and G. López Acedo, Lower bounds for normal structure coefficients, Proc. Roy. Soc. Edinburgh. Sect. A 121 (1992), 245-252.

8. K. Goebel and T. Sekowski, The modulus of noncompact convexity, Ann. Univ. Marie Curie Sklodowska. Sect A XXXVIII, 3 (1984), 41-48.

9. R. Huff, Banach spaces which are nearly uniformly convex, Rocky Mountain J. Math. 4 (1980), 743-749.

10. J. P. Partington, On nearly uniformly convex Banach spaces, Math. Proc. Camb. Phil. Soc. 93 (1983), 127-129. $535-545$

11. S. Prus, On Bynum's fixed point theorem, Atti. Sem. Mat. Fis. Univ. Modena 38 (1990),

12. S. Prus, Nearly uniformly smooth Banach spaces, Bollettino U.M.I. 3(7) (1989), 507-521.

13. T. Sekowski and A. Stachura, Noncompact smoothness and noncompact convexity, Atti. Sem. Mat. Fis. Univ. Modena 36 (1988), 329-338.

\author{
Departamento de Ańalisis Matemático \\ UNIVERSIDAD DE SEVILLA \\ APDO. 1160 \\ 41080-SEVILLA \\ SPAIN
}

